\title{
Degradation of methylene blue organic wastewater by plasma assisted method
}

\author{
Zhuansun Menglin ${ }^{1}$, Liu Linsheng ${ }^{2 *}$, Wang Yanting ${ }^{3}$, Zhai Limin ${ }^{4}$, Chen Xinyi ${ }^{5}$, Tian Chunsuo ${ }^{6}$ \\ ${ }^{1}$ Zhuansun Menglin, College of Electronic Engineering, Guangxi Normal University, China \\ ${ }^{2 *}$ Liu Linsheng, College of Electronic Engineering, Guangxi Normal University, China \\ ${ }^{3}$ Wang Yanting, College of Electronic Engineering, Guangxi Normal University, China \\ ${ }^{4}$ Zhai Limin, College of Electronic Engineering, Guangxi Normal University, China \\ ${ }^{5}$ Chen Xinyi, College of Electronic Engineering, Guangxi Normal University, China \\ ${ }^{6}$ Tian Chunsuo, College of Electronic Engineering, Guangxi Normal University, China
}

\begin{abstract}
In the current situation of water shortage and serious pollution, plasma discharged technology has a useful application prospect in sewage treatment. In order to control the degradation rate of pollutants, the effects of different gas environment, $\mathrm{OH}$ scavenger ethanol and $\mathrm{H}_{2} \mathrm{O}_{2}$ on the degradation of methylene blue simulated organic wastewater by plasma assisted method were studied. The experimental results show that the effect of air plasma discharge is slightly better than that of atmospheric pressure argon discharge. Ethanol can remove $\mathrm{OH}$, thus reducing the degradation rate of methylene blue, while $\mathrm{H}_{2} \mathrm{O}_{2}$ can improve the degradation rate.
\end{abstract}

\section{Introduction}

At present, human beings are facing the problem of shortage of fresh water resources. With the rapid development of modern industry, water pollution has become a new reason for the lack of water resources. In recent years, the situation of sewage treatment in China is more severe, such as large amount of sewage disposal, low treatment efficiency and disorderly discharge. Water resources is polluted or damaged. The problem of dye wastewater is extremely serious. It is difficult to decolorize because of its deep color, high content of organic pollutants, complex components and high biological toxicity. In addition, the dyes have antiphotolysis and anti-oxidation properites, which are difficult to be effectively degraded by conventional methods, causing serious pollution of the water environment.

The traditional water treatment methods are needed to renew materials, the cost is high, and sometimes chemical reagents are used, which may cause water pollution again ${ }^{[1]}$. With the demand for new methods of water treatment, advanced oxidation technology began to appear. When advanced oxidation technology is applied to water treatment, it can produce strong oxidizing substances in water, and can effectively purify sewage, so it has a good application prospect ${ }^{[2]}$. There are many types of advanced oxidation methods, such as Fenton reaction, photocatalytic oxidation and electrochemical oxidation. Fenton reaction and photocatalytic oxidation need chemical reagents, which inevitably lead to secondary pollution and expensive reagents. Electrochemical advanced oxidation technique is a green and efficient water treatment method, which is based on the generation of hydroxyl radicals by electron transfer. The advantage of electrochemical oxidation method is that only a little or no chemical reagents are needed, and organic pollutants can be treated more quickly ${ }^{[3]}$.

Plasma discharge technique combines the advantages of advanced oxidation methods. The inelastic collision between high-energy electrons and water in the process of plasma discharge leads to a series of complicated processes such as excitation, dissociation and ionization, and a large number of active free radicals and strong oxidizing molecules are produced. Among them, the most important one is $\mathrm{OH}$, which is second only to fluorine in oxidizing properties. It can destroy the C-C bond of organic matters and form $\mathrm{C}-\mathrm{OH}$ bond in the process of reaction with organic matters, which will destroy the structures of organic matters ${ }^{[4]}$.

In this study, atmospheric pressure glow discharge plasma was used to degrade methylene blue to simulate the degradation process of organic wastewater. The degradation rate of methylene blue by plasma was investigated in detail by adding additives and changing discharge gas environment.

\section{Experimental Setup And Principle}

\subsection{Experimental setup and degradation principle}

Plasma is an ionized gaseous substance composed of atoms and positive and negative particles, which can be produced by different types of high voltage discharge.

*xxx: author@e-mail.org Liu Linsheng: linshengliu@163.com 
When the plasma acts on the aqueous solution, a large number of active substances, such as $\mathrm{OH}, \mathrm{O}_{3}, \mathrm{O}$ and $\mathrm{e}_{\mathrm{aq}}{ }^{-}$, will be produced in the liquid ${ }^{[5]}$. The process of water treatment by plasma technique includes many types of advanced oxidation processes, such as ozone oxidation, UV-photocatalytic oxidation and pyrolysis. It is a comprehensive and environmentally friendly water treatment technique. Organic pollutants in the water can be rapidly degraded by the oxidation and reduction of the substances, or in the synergistic effect, so as to achieve the purpose of sewage purification ${ }^{[6]}$.

The experimental cycle setup is shown in Figure 1. The high voltage DC power supply(LAS $20 \mathrm{kV}, 50 \mathrm{~mA}$ ) was used to discharge at the tungsten steel electrode, which located $3 \mathrm{~mm}$ above the flowing solution surface on the top of the plasma reactor, and plasma was generated between the liquid surface and the electrode. Argon (Ar) is sprayed from the conical nozzle of tungsten steel electrode at a flow rate of 20 SCCM through the gas mass flow controller(D07-19B).

The circulation process of the reaction solution is as follows: firstly, the solution is pumped into the blending bottle in the water bath by the peristaltic pump (RZ1030) through the silicone tube, mixed by magnetic stirring (DFY-5L/10, water temperature controlled at $25^{\circ} \mathrm{C}$ ), and then flows into the quartz cuvette $(10 \mathrm{~mm}$ in optical lengthth) to monitor the absorption spectrum in real time. Then the solution flows from the cuvette into the plasma circulation reactor, and flows from the bottom of the reactor to the top to react with the plasma. Finally, the solution reacts with the plasma, and then returns to the peristaltic pump for the next cycle. The flow of liquid and magnetic stirring can promote the diffusion of organic dyes, so that organic dyes can be uniformly degraded.

The light source used for the experiment is tungsten halogen lamp (DH-2000), and a micro fiber spectrometer (USB6500 Pro) is used for real-time monitoring. The experimental setup is simple and the experimental cost is low.

The volume of the solution was $80 \mathrm{ml}$, the concentration was $5 \mathrm{mg} / \mathrm{L}$, the flow rate of the peristaltic pump was $50 \mathrm{ml} / \mathrm{min}$, and the reagent used was methylene blue (1\%, Aladdin).

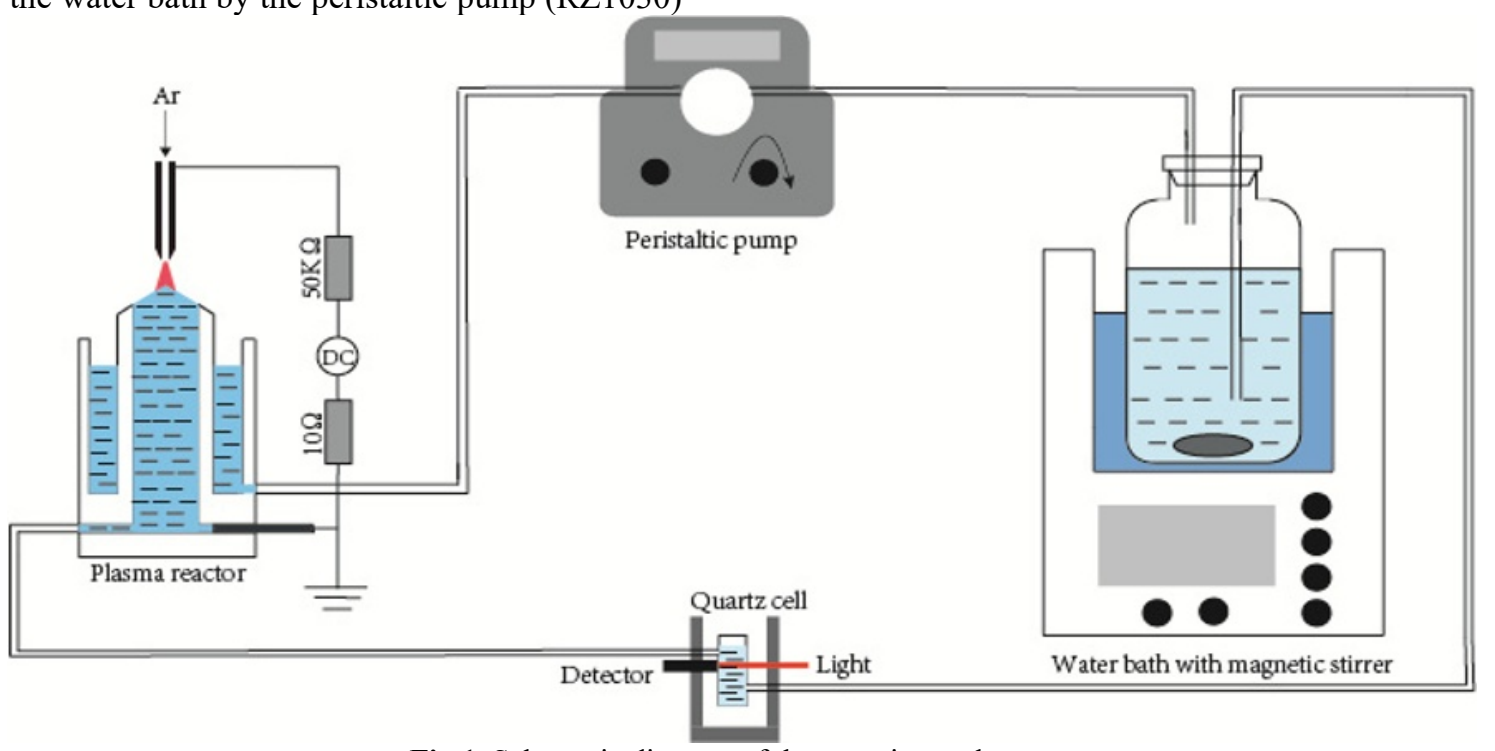

Fig.1. Schematic diagram of the experimental setup.

According to the bond dissociation energy(BDE) theory, the lower the $\mathrm{BDE}$ is, the more active the chemical bond is and the easier it is for old bonds to break and new bonds to form. The molecular structure of methylene blue is shown in the Figure 2, the main chromogenic group is '-S-'. '9C-S' and ' $10 \mathrm{C}-\mathrm{S}$ ' are easily broken by oxidation ${ }^{[7]}$.<smiles></smiles>

Fig.2. Molecular diagram of methylene blue

\subsection{Methylene blue concentration monitoring}

Methylene blue solution has a maximum absorption peak at about $660 \mathrm{~nm}$, and this is an isochromatic absorption point. The absorption intensity at the isochromatic absorption point is only related to the concentration of methylene blue in the solution. Therefore, the absorbance at this point can represent the relative concentration of methylene blue in solution. The change of absorbance with time can reflect the decomposition rate of methylene blue. The UV Vis absorption spectra of the solution was measured by spectrometer(USB6500 Pro), and the light source was tungsten halogen lamp(DH-2000). All the absorption spectra were normalized.

\subsection{Decolorization rate of methylene blue}

The absorbance of methylene blue after degradation was measured by optical fiber spectrometer, and the corresponding concentration value was calculated. The 
degradation rate of methylene blue was calculated according to the following formula.

$$
\eta=\frac{C_{0}-C_{1}}{C_{0}} \times 100 \%
$$

$\eta$ represents the removal rate of methylene blue.

$C_{0}$ represents the initial concentration, $\mathrm{mg} / \mathrm{L}$.

$C_{1}$ represents the degraded concentration, $\mathrm{mg} / \mathrm{L}$.

Through this formula, we can directly show the removal rate of methylene blue.

\section{Results and discussion}

\subsection{Gas environment}

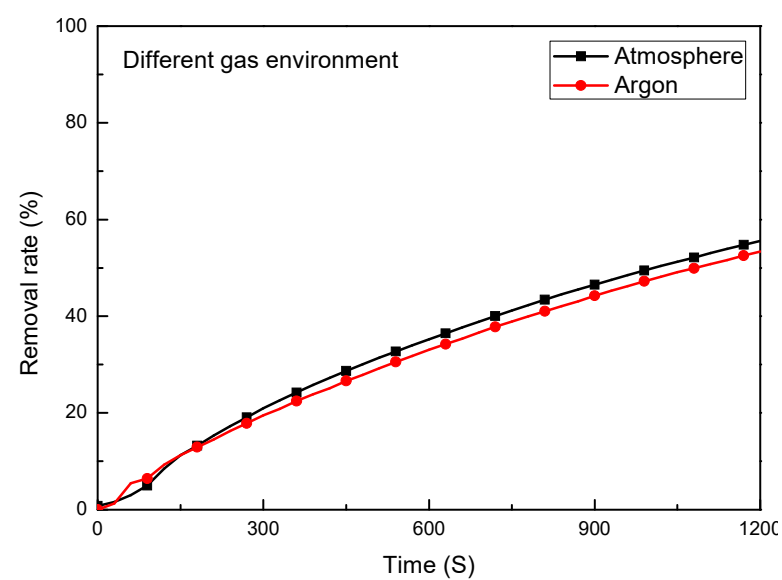

Fig.3.1 Decolorization rate of methylene blue in air and argon gas environment, $30 \mathrm{~mA}$ current, 20 min reaction time

In this experiment, the gas environment of plasma is changed. Atmospheric pressure argon discharge and air discharge were used. As shown in Fig. 3.1, the decomposition rate of air discharge was slightly higher than that of atmospheric pressure argon discharge. In the case of air discharge, $\mathrm{HNO}_{3}$ and $\mathrm{HNO}_{2}$ were formed in the solution due to the presence of a large amount of $\mathrm{N}_{2}$ and $\mathrm{O}_{2}$. As a result, the $\mathrm{PH}$ value of the solution decreases and $\mathrm{ONOO}^{-}$was formed, which plays an auxiliary role in oxidation. Therefore, the decomposition rate was slightly faster than that of argon discharge at atmospheric pressure ${ }^{[8]}$.

\subsection{Adding ethanol}

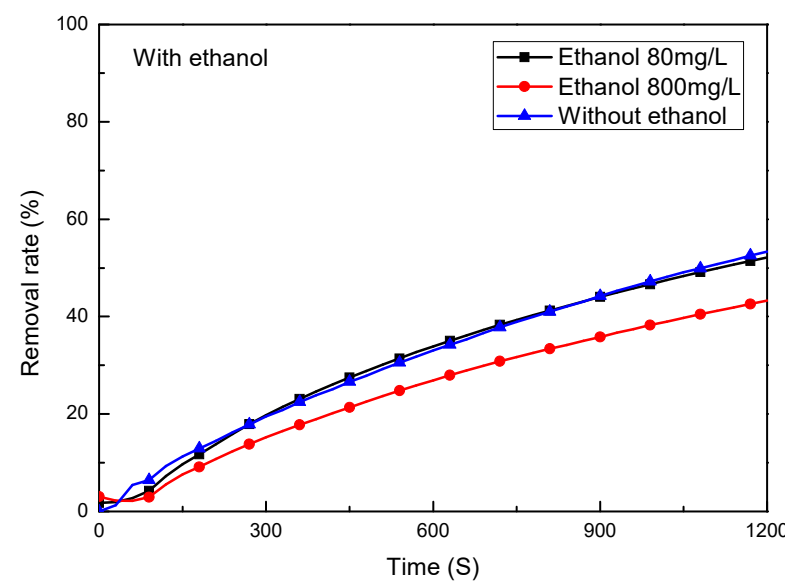

Fig.3.2 Decolorization rate of methylene blue with $80 \mathrm{mg} / \mathrm{L}$ and $800 \mathrm{mg} / \mathrm{L}$ concentration of ethanol, $30 \mathrm{~mA}$ current, $20 \mathrm{~min}$ reaction time

Among a series of active substances generated in the contact reaction between plasma and liquid surface, $\mathrm{OH}$ as a strong oxidant, played an important role in the process of oxidative degradation of methylene blue. Ethanol can provide electrons to react with $\mathrm{OH}$ to act as $\mathrm{OH}$ scavenger. The addition of ethanol decreased the concentration of $\mathrm{OH}$ and the decomposition rate of methylene blue. As shown in Figure 3.2, when low concentration ethanol was added, the degradation rate decreased slightly after 15 minutes. When the concentration of ethanol improved, the degradation rate of methylene blue decreased significantly ${ }^{[9]}$.

\subsection{Adding $\mathrm{H}_{2} \mathrm{O}_{2}$}

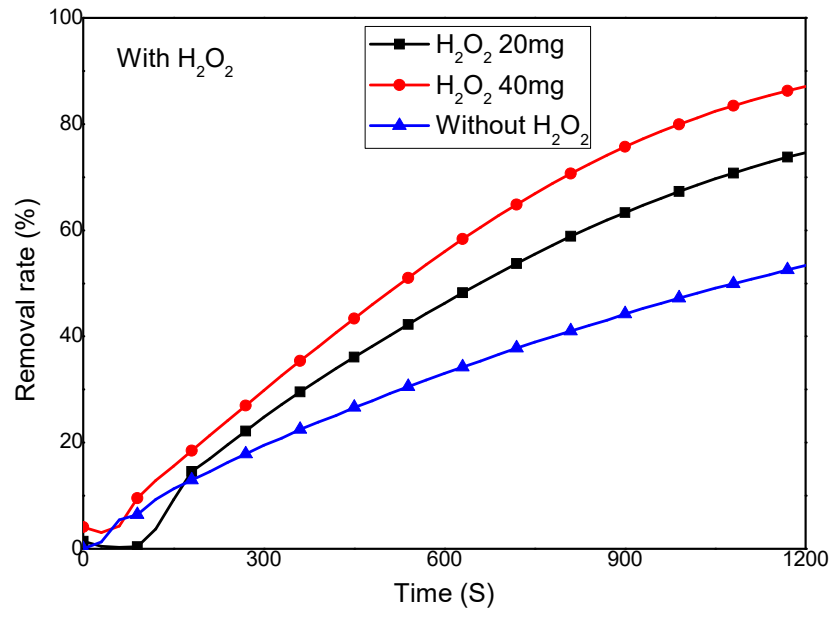

Fig.3.3 Decolorization rate of methylene blue with $20 \mathrm{mg} / \mathrm{L}$ and $40 \mathrm{mg} /$ Lconcentration of $\mathrm{H}_{2} \mathrm{O}_{2}, 30 \mathrm{~mA}$ current, $20 \mathrm{~min}$ reaction time

When hydrogen peroxide was added, hydrogen peroxide and most organic compounds will not react significantly, but because of its existence, it can react with other substances in different ways to produce more $\mathrm{OH}$. Thus, the degradation of methylene blue was accelerated $^{[10]}$.

$$
\begin{gathered}
\mathrm{H}_{2} \mathrm{O}_{2} \rightarrow \mathrm{H}^{+}+\mathrm{HO}_{2}^{-} \\
\mathrm{O}_{3}+\mathrm{HO}_{2}^{-} \rightarrow \mathrm{O}_{2}^{-}+\cdot \mathrm{OH}^{-} \mathrm{O}_{2}
\end{gathered}
$$


It can be concluded from this group of experiments that $\mathrm{H}_{2} \mathrm{O}_{2}$ is a very important material in the experiment of methylene blue degradation by plasma method.

\section{Conclusion}

The degradation rate of methylene blue in air plasma discharge was slightly higher than that in argon plasma discharge. When ethanol was added into the solution, the $\mathrm{OH}$ concentration in the solution could be reduced, and the degradation rate of methylene blue could be reduced. When $\mathrm{H}_{2} \mathrm{O}_{2}$ was added, $\mathrm{H}_{2} \mathrm{O}_{2}$ generates more $\mathrm{OH}$ through the reaction to accelerate the degradation rate of methylene blue. Plasma degradation of organic pollutants in-depth study, can further improve the treatment efficiency, has a good application prospect, and provides an important safeguard for the treatment of sewage.

\section{Acknowledgments}

This work was supported by the doctoral scientific research fund of Guangxi Normal University (Grant No. 2017BQ019); Innovation Project of Guangxi Graduate Education(Grant No. JGY2020024); Special project of Guangxi Normal University for cultivating students' scientific research ability(Natural Science): (Grant No.2019YR007).

\section{References}

1. SONUNE A, GHATE R. Developments in wastewater treatment methods[J]. Desalination, 2004, 167(1):55-63.

2. Glaze W H, Kang J W, Chapin D H. The Chemistry of Water Treatment Processes Involving Ozone, Hydrogen Peroxide and Ultraviolet Radiation[J]. Ozone ence \& Engineering, 1987, 9(4):335-352.

3. Nidheesh P V, Gandhimathi R. Trends in electroFenton process for water and wastewater treatment: An overview[J]. Desalination, 2012, 299:1-15.

4. Lukes P. Water treatment by pulsed streamer corona discharge[D]. Prague: Institue of Chemical Technology, 2001.

5. Sarangapani C, Dixit Y, Milosavljevic V, et al. Optimization of atmospheric air plasma for degradation of organic dyes in wastewater[J]. Water Science \& Technology, 2017, 75(1):207-219.

6. S. Ma, K. Kim, S. Chun, S. Y. Moon, and Y. Hong, Plasma-assisted advanced oxidation process by a multi-hole dielectric barrier discharge in water and its application to wastewater treatment[J], Chemosphere, 2020, 243:125377.

7. Huang F, Chen L, Wang H, et al. Analysis of the degradation mechanism of methylene blue by atmospheric pressure dielectric barrier discharge plasma[J]. Chemical Engineering Journal, 2010, 162(1):250-256.
8. Bangbang H, Xinning G, Xin W, et al. What Are the Effective Reactants in the Plasma-Induced Wastewater Treatment[J]. Journal of the Electrochemical Society, 2018, 165(10):E454-E459.

9. Z. Shen, Y. Zhou, and J. Wang, Bioresour. Technol., 2013, 131:33.

10. Lukes P, Dolezalova E, Sisrova I, et al. Aqueousphase chemistry and bactericidal effects from an air discharge plasma in contact with water: evidence for the formation of peroxynitrite through a pseudosecond-order post-discharge reaction of $\mathrm{H}_{2} \mathrm{O}_{2}$ and $\mathrm{HNO}_{2}[\mathrm{~J}]$. Plasma Sources ence \& Technology, 2014, 23(1):015019. 